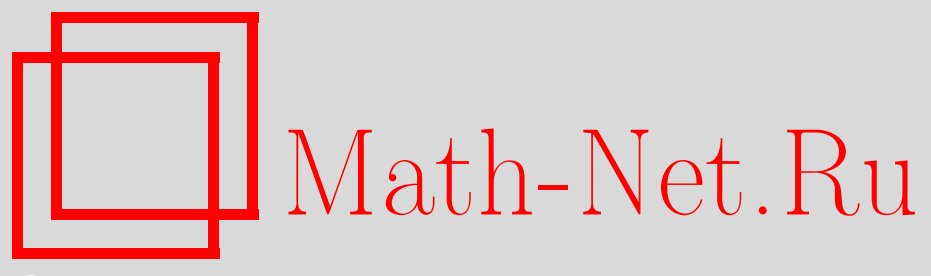

В. А. Кыров, Алгебра Ли группы движений феноменологически симметричной геометрии, Матем. заметки, 2012, том 91, выпуск 2, 312-315

DOI: https://doi.org/10.4213/mzm8486

Использование Общероссийского математического портала Math-Net.Ru подразумевает, что вы прочитали и согласны с пользовательским соглашением http://www . mathnet.ru/rus/agreement

Параметры загрузки:

IP : 54.237 .59 .107

26 апреля 2023 г., 12:56:03

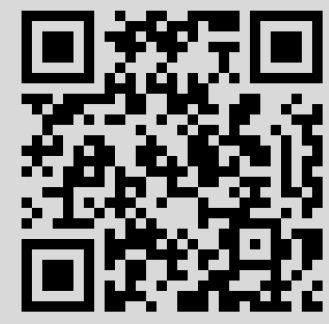




\section{Алгебра Ли группы движений феноменологически симметричной геометрии}

\section{В. А. Кыров}

1. Введение. Рассмотрим многообразие $M, \operatorname{dim} M=n$. Пусть на $M$ задана гладкая функция $f: M \times M \rightarrow R$, называемая метрической, с открытой и плотной в $M \times M$ областью определения $S_{f}$. Значение метрической функции обозначается $f(i j)$, где $\langle i, j\rangle \in S_{f}$. Локальные координаты в $M$ обозначим через $\left(x_{1}, \ldots, x_{n}\right)$. Пусть выполняются следующие аксиомы [1].

АКСИомА невЫРОЖДЕНности. Для любого упорядоченного набора $n+1$ точек $\left\langle i, i_{1}\right.$, $\left.\ldots, i_{n}\right\rangle$ из открытого и плотного подмножества в $M^{n+1}$ таких, что

$$
\left\langle i, i_{1}\right\rangle, \ldots,\left\langle i, i_{n}\right\rangle \in S_{f}, \quad\left\langle i_{1}, i\right\rangle, \ldots,\left\langle i_{n}, i\right\rangle \in S_{f},
$$

выполняются неравенства

$$
\frac{\partial\left(f\left(i i_{1}\right), \ldots, f\left(i i_{n}\right)\right)}{\partial\left(x_{1 i}, \ldots, x_{n i}\right)} \neq 0, \quad \frac{\partial\left(f\left(i_{1} i\right), \ldots, f\left(i_{n} i\right)\right)}{\partial\left(x_{1 i}, \ldots, x_{n i}\right)} \neq 0,
$$

где $\left(x_{1 i}, \ldots, x_{n i}\right)$ - координаты точки $i \in M$.

АКСИОМА ФЕНОМЕНОЛОГИЧЕСКОЙ СИмМЕТРИИ. Для некоторой окрестности любой последовательности точек $\left\langle i_{1}, \ldots, i_{n+2}\right\rangle$ из открытого и плотного подмножества прямого произведения $M^{n+2}$ такой, что

$$
\left\langle i_{p}, i_{q}\right\rangle \in S_{f}, \quad p, q=1, \ldots, n+2, \quad p \neq q,
$$

имеет место тождество

$$
\Phi\left(f\left(i_{1} i_{2}\right), \ldots, f\left(i_{n+1} i_{n+2}\right)\right)=0, \quad \operatorname{grad} \Phi \neq 0 .
$$

ОПределЕниЕ 1 . Говорят, что на многообразии $M$ метрическая функция $f$ задает феноменологически симметричную геометрию, если выполняются аксиомы невырожденности и феноменологической симметрии.

ОПределЕниЕ 2. Локальный диффеоморфизм $\lambda: M \rightarrow M$ называется локалъным движением, если для любой пары $\langle i, j\rangle \in S_{f}$ такой, что $\langle\lambda(i), \lambda(j)\rangle \in S_{f}$, имеет место равенство $f(\lambda(i), \lambda(j))=f(i j)$.

Множество всех движений образует группу Ли. Можно показать, что размерность группы движений максимальна и равна $n(n+1) / 2$. Доказывается, что двухточечным инвариантом такой группы, по которому она восстанавливается [1], является метрическая функция.

В данной работе группа движений рассматривается локально, поэтому многообразие $M$ можно отождествить с $\mathbb{R}^{n}$. Группу движений обозначим $G\left(\mathbb{R}^{n}\right)$, она имеет размерность $n(n+1) / 2$. Базисные операторы алгебры Ли $L$ группы движений обозначим $X_{1}, \ldots$, $X_{n(n+1) / 2}$. Так как $f(i j)$ - двухточечный инвариант группы движений, то согласно критерию локальной инвариантности выполняется система уравнений [2; с. 35]

$$
X_{\mu}(i) f(i j)+X_{\mu}(j) f(i j)=0, \quad \mu=1, \ldots, \frac{n(n+1)}{2} .
$$

В локальных координатах $\left(x_{1}, \ldots, x_{n}\right)$ для базисных операторов имеем

$$
X_{\mu}=\lambda_{\mu}^{1}\left(x_{1}, \ldots, x_{n}\right) \partial_{x_{1}}+\cdots+\lambda_{\mu}^{n}\left(x_{1}, \ldots, x_{n}\right) \partial_{x_{n}},
$$

где $\lambda_{\mu}^{1}\left(x_{1}, \ldots, x_{n}\right), \ldots, \lambda_{\mu}^{n}\left(x_{1}, \ldots, x_{n}\right)$ - достаточно гладкие функции.

Основная цель данной работы - это доказательство следующих теорем.

(C) B. А. Кыров, 2012 
Tеорема 1. В некоторой окрестности $U(i) \subset \mathbb{R}^{n}$ точки $i$ группа Ли двиюений $G\left(\mathbb{R}^{n}\right)$ феноменологически симметричной геометрии транзитивна.

Теорема 2. Алгебру Ли L группы движений феноменологически симметричной геометрии нельзя представить в виде прямой суммы двух идеалов.

\section{2. Доказательство теорем.}

ДокАЗАТЕЛЬСТво тЕОРЕмы 1 . При $n=1$ теорема очевидна. Проведем доказательство при $n \geqslant 2$. Пусть группа движений $G\left(\mathbb{R}^{n}\right)$ с невырожденным двухточечным инвариантом $f$ в некоторой окрестности $V(i)$ точки $i \in \mathbb{R}^{n}$ интранзитивна. Тогда инвариантное многообразие этой группы имеет размерность $m$, причем при $n=2 m=1$, а при $n \geqslant 3$ $m=2, \ldots, n-1$. В окрестности точки $i$ локальные координаты введем так, чтобы $m$ координатных линий принадлежали инвариантному многообразию. Пусть $U(i)$ пересечение введенной координатной окрестности с $V(i)$, а $M(i)$ - пересечение $U(i)$ с инвариантным многообразием, проходящим через точку $i$. Координаты на $M(i)$ обозначим $x_{1}, \ldots, x_{m}$. В этих координатах для компонент операторов (2) имеем

$$
\lambda_{\mu}^{\nu}=\lambda \nu_{\mu}\left(x_{1}, \ldots, x_{m}\right), \quad \lambda_{\mu}^{\omega}=0,
$$

где $\mu=1, \ldots, n(n+1) / 2, \nu=1, \ldots, m, \omega=m+1, \ldots, n$. Решая систему (1) для найденных операторов, получаем вырожденный инвариант

$$
f(i j)=f\left(x_{m+1}(i), \ldots, x_{n}(i), x_{m+1}(j), \ldots, x_{n}(j)\right) .
$$

Противоречие.

ДоКАЗАТЕЛЬСтво теОРемЫ 2. Очевидно, $n \geqslant 2$. ПредПоложим, что $L=J_{1} \oplus J_{2}$, где $J_{1}$, $J_{2}$ - идеалы. Рассмотрим группы движений $G\left(U(i), J_{1}\right)$ и $G\left(U(i), J_{2}\right)$ с алгебрами Ли $J_{1}$ и $J_{2}$. Им соответствуют инвариантные многообразия $M\left[G\left(U(i), J_{1}\right)\right]$ и $M\left[G\left(U(i), J_{2}\right)\right]$. Выделим следующие случаи:

I) $\operatorname{dim} J_{1}=1, \operatorname{dim} J_{2}=n(n+1) / 2-1$,

II) $\operatorname{dim} J_{1}=m, \operatorname{dim} J_{2}=n(n+1) / 2-m, m \geqslant 2$.

Лемма. Если алгебра Ли группы движений феноменологически симметричной геометрии разлагается в прямую сумму двух идеалов $L=J_{1} \oplus J_{2}$, то инвариантные многообразия групп с алгебрами Ли $J_{1}$ и $J_{2}$ пересекаются транверсально.

ДокАЗАтельство. Рассмотрим алгебру Ли группы движений феноменологически симметричной геометрии $L=J_{1} \oplus J_{2}$, где $J_{1}$ и $J_{2}$ - ее идеалы [2; с. 180]. Пусть $G(U(i))-$ группа Ли преобразований окрестности $U(i) \subset \mathbb{R}^{n}$ точки $i$ с алгеброй Ли $L, G\left(U(i), J_{1}\right)$ и $G\left(U(i), J_{2}\right)$ - ее подгруппы Ли с алгебрами Ли $J_{1}$ и $J_{2}$, а $M\left[G\left(U(i), J_{1}\right)\right]$ и $M\left[G\left(U(i), J_{2}\right)\right]-$ инвариантные многообразия в $U(i)$. Предположим, что эти многообразия пересекаются в $j \in U(i)$. Касательные пространства в $j$ к инвариантным многообразиям обозначим $T_{j}\left(M\left[G\left(U(i), J_{1}\right)\right]\right)$ и $T_{j}\left(M\left[G\left(U(i), J_{2}\right)\right]\right)$. Произвольный вектор из $T_{j}\left(M\left[G\left(U(i), J_{1}\right)\right]\right)$ является линейной комбинацией векторов, индуцированных операторами из $J_{1}$ в $j$. Аналогично и для касательного пространства $T_{j}\left(M\left[G\left(U(i), J_{2}\right)\right]\right)$. Из свойства транзитивности группы движений $G(U(i))$ получаем

$$
T_{j}(U(i))=T_{j}\left(M\left[G\left(U(i), J_{1}\right)\right]\right) \oplus T_{j}\left(M\left[G\left(U(i), J_{2}\right)\right]\right) .
$$

УтверЖДЕниЕ. Если два оператора алгебры Ли L группы движений феноменологически симметричной геометрии коммутируют, то их интегральные кривые в некоторой окрестности $U(i)$ точки $i \in \mathbb{R}^{n}$ пересекаются трансверсально. 
Действительно. Пусть $X$ и $Y$ - коммутативные операторы, т.е. $[X, Y]=0$. Тогда можно ввести такую систему локальных координат в $U(i)$, относительно которой

$$
X=\partial_{x_{1}}, \quad Y=Y_{1}\left(x_{2}, \ldots, x_{n}\right) \partial_{x_{1}}+Y_{2}\left(x_{2}, \ldots, x_{n}\right) \partial_{x_{2}}+\cdots+Y_{n}\left(x_{2}, \ldots, x_{n}\right) \partial_{x_{n}}
$$

(см. $\left[2 ;\right.$ с. 40]). Если $Y_{2}^{2}+\cdots+Y_{n}^{2}=0$, то $X=\partial_{x}$ и $Y=Y_{1}\left(x_{2}, \ldots, x_{n}\right) \partial_{x} ;$ следовательно двухточечный инвариант группы движений $G\left(\mathbb{R}^{n}\right)$ имеет вид $f(i j)=f\left(x_{2}(i), \ldots, x_{n}(i)\right.$, $\left.x_{2}(j), \ldots, x_{n}(j)\right)$, т.е. вырожден. Противоречие. Пусть теперь $Y_{2}^{2}+\cdots+Y_{n}^{2} \neq 0$. Тогда в $U(i)$ интегральные кривые пересекаются трансверсально.

Вернемся к доказательству леммы. Из определения прямой суммы идеалов следует, что $[X, Y]=0$ для любых $X \in J_{1}$ и $Y \in J_{2}$. Тогда по выше доказанному утверждению $M\left[G\left(U(i), J_{1}\right)\right]$ и $M\left[G\left(U(i), J_{2}\right)\right]$ могут пересекаться только трансверсально в $U(i)$.

I) Пусть $\operatorname{dim} J_{1}=1, \operatorname{dim} J_{2}=n(n+1) / 2-1$. Тогда

$$
\operatorname{dim} M\left[G\left(U(i), J_{1}\right)\right]=1, \quad \operatorname{dim} M\left[G\left(U(i), J_{2}\right)\right]=n-1 .
$$

Из леммы следует, что в $U(i)$ можно ввести такие координаты, чтобы первая из них являлась локальной координатой на $M\left[G\left(U(i), J_{1}\right)\right]$, которую обозначим $x_{1}$, а остальные локальными координатами на $M\left[G\left(U(i), J_{2}\right)\right]: x_{2}, \ldots, x_{n}$. Тогда для базисных операторов алгебры $L$ имеем

$$
X_{1}=\partial_{x_{1}}, \quad X_{\mu}=\lambda_{\mu}^{2}\left(x_{2}, \ldots, x_{n}\right) \partial_{x_{2}}+\cdots+\lambda_{\mu}^{n}\left(x_{2}, \ldots, x_{n}\right) \partial_{x_{n}},
$$

где $\mu=2, \ldots, n(n+1) / 2$. Поэтому двухточечный инвариант равен $f(i j)=f\left(g\left(x_{2}(i), \ldots\right.\right.$, $\left.\left.x_{n}(j), x_{2}(j), \ldots, x_{n}(j)\right)\right)$, т.е. вырожден. Противоречие.

II) Пусть $\operatorname{dim} J_{1}=m, \operatorname{dim} J_{2}=n(n+1) / 2-m$. Тогда

$$
\operatorname{dim} M\left[G\left(U(i), J_{1}\right)\right]=k, \quad \operatorname{dim} M\left[G\left(U(i), J_{2}\right)\right]=n-k,
$$

причем $k \leqslant m, n-k \leqslant n(n+1) / 2-m$. Рассуждая как и выше, приходим к вырожденному двухточечному инварианту. Противоречие. Теорема 2 доказана полностью.

СлЕДСтвиЕ. Если алгебра Ли L группы движений феноменологически симметричной геометрии полупроста, то она проста.

Это утверждение вытекает из теоремы 2 и структурной теоремы [2; с. 191]: конечномерная алгебра Ли L полупроста тогда и только тогда, когда существует разложение $L=L_{1} \oplus \cdots \oplus L_{k}$, где $L_{s}-$ идеалы, являюшиеся простыми подалгебрами $Л и, s=1, \ldots, k$.

3. Примеры. Рассмотрим форму Киллинга алгебра Ли $L$ [2; с. 190]:

$$
K[X, Y]=\operatorname{tr}(\operatorname{ad}(X) \circ \operatorname{ad}(Y)),
$$

где $X, Y \in L, \operatorname{ad}(X): L \rightarrow L-$ присоединенный оператор. Напомним также критерий полупростоты [2; с. 191]: алгебра Ли L полупроста тогда и толъко тогда, когда ее форма Киллинга невырождена.

Пространство Евклида. Известно, что форма Киллинга алгебры Ли группы движений простраства Евклида вырождена и поэтому алгебра Ли не является полупростой.

Пространство Гельмгольца. Метрическая функция [3]

$$
f(i j)=\left[\left(x_{i}-x_{j}\right)^{2}+\left(y_{i}-y_{j}\right)^{2}\right] \exp \left\{2\left(\gamma \operatorname{arctg}\left(\frac{y_{i}-y_{j}}{x_{i}-x_{j}}\right)+z_{i}+z_{j}\right)\right\} .
$$


Коммутционные соотношения базисных операторов алгебры Ли ее группы движений [3]:

$$
\begin{gathered}
{\left[X_{1}, X_{2}\right]=0, \quad\left[X_{1}, X_{3}\right]=-\gamma X_{1}+X_{2}, \quad\left[X_{1}, X_{4}\right]=-X_{1}-\gamma X_{2},} \\
{\left[X_{1}, X_{5}\right]=2 X_{4}, \quad\left[X_{1}, X_{6}\right]=2 X_{3}, \quad\left[X_{2}, X_{3}\right]=-X_{1}-\gamma X_{2},} \\
{\left[X_{2}, X_{4}\right]=\gamma X_{1}-X_{2}, \quad\left[X_{2}, X_{5}\right]=-3 X_{3}, \quad\left[X_{2}, X_{6}\right]=2 X_{4},} \\
{\left[X_{3}, X_{4}\right]=0, \quad\left[X_{3}, X_{5}\right]=-X_{6}-\gamma X_{5}, \quad\left[X_{3}, X_{6}\right]=X_{5}-\gamma X_{6},} \\
{\left[X_{4}, X_{5}\right]=\gamma X_{6}-X_{5}, \quad\left[X_{4}, X_{6}\right]=-\gamma X_{5}-X_{6}, \quad\left[X_{5}, X_{6}\right]=0 .}
\end{gathered}
$$

Форма Киллинга имеет вид

$$
\begin{gathered}
K[X, Y]=4\left(\gamma^{2}-1\right)\left(X^{3} Y^{3}-X^{4} Y^{4}\right)+8 \gamma\left(X^{3} Y^{4}+X^{4} Y^{3}\right)+8\left(X^{1} Y^{5}+X^{5} Y^{1}\right) \\
+8 \gamma\left(X^{1} Y^{6}+X^{6} Y^{1}\right)-8 \gamma\left(X^{2} Y^{5}+X^{5} Y^{2}\right)+8\left(X^{2} Y^{6}+X^{6} Y^{2}\right) .
\end{gathered}
$$

Доказывается невырожденность формы Киллинга, поэтому алгебра Ли полупроста. По следствию из теоремы 2 эта алгебра Ли проста.

Сферическое пространство. Невырожденность формы Киллинга для алгебры Ли группы движений сферического пространства, т.е. группы вращений, является хорошо известным фактом. Ее алгебра Ли проста.

\section{СПИСОК ЦИТИРОВАННОЙ ЛИТЕРАТУРЫ}

[1] Г. Г. Михайличенко, Докл. АН СССР, 269:2 (1983), 284-288. [2] Л. В. Овсянников, Групповой анализ дифференииальных уравнений, Наука, М., 1978. [3] В. А. Кыров, "Шестимерные алгебры Ли групп движений трехмерных феноменологически симметричных геометрий": Г. Г. Михайличенко, Полиметрические геометрии, Изд-во Новосибирск. гос. ун-та, Новосибирск, 2001, 116-143.

В. А. Кыров

Поступило

Горно-алтайский государственный университет

22.05 .2009

E-mail: kfizika@gasu.ru

Исправленный вариант

14.03.2011 